\title{
Sympathectomy via a posterior approach after a failed trans-thoracic approach: a case of its use for arrhythmia
}

\author{
Daniel Blatt, BS, ${ }^{1}$ Barry Cheaney II, BS, ${ }^{1}$ Katherine Holste, MD, ${ }^{2}$ \\ Seshadri Balaji, MBBS, MRCP(UK), PhD, ${ }^{4}$ and Ahmed M. Raslan, $\mathrm{MD}^{3}$ \\ ${ }^{1}$ School of Medicine, Oregon Health \& Science University, Portland, Oregon; ${ }^{2}$ Department of Neurosurgery, University of \\ Michigan, Ann Arbor, Michigan; ${ }^{3}$ Department of Neurological Surgery and ${ }^{4}$ Division of Cardiology, Department of Pediatrics, \\ Oregon Health \& Science University, Portland, Oregon
}

\begin{abstract}
OBJECTIVE Congenital long QT syndrome (LQTS) provides an opportunity for neurosurgical intervention. Medication and implantable cardiac defibrillator (ICD)-refractory patients often require left cardiac sympathetic denervation (LCSD) via anterior video-assisted thoracoscopic surgery (VATS). However, this approach has major pulmonary contraindications and risks, with a common concern in children being their inability to tolerate single-lung ventilation. At Oregon Health \& Science University, the authors have developed a posterior approach—extrapleural, minimally invasive, T1-5 LCSD_that minimizes this risk.
\end{abstract}

METHODS A 9-year-old girl with LQTS type III presented to the emergency department while experiencing ventricular tachycardia (VT) and ventricular fibrillation (VF) with multiple ICD firings. Medical management failed to resolve the VF/ VT. VATS was attempted but could not be safely performed due to respiratory insufficiency. The patient was reintubated for dual-lung ventilation and repositioned prone. Her respiratory insufficiency resolved. Using METRx serial dilating tubes under the microscope, the left T1-5 sympathetic ganglia were sectioned and removed.

RESULTS Postoperatively, the patient had no episodes of VF/VT, pneumothorax, hemothorax, or Horner syndrome. With mexiletine and propranolol, she has remained largely VF/VT free, with only one VT episode during the 2-year follow-up period.

CONCLUSIONS Minimally invasive, posterior, extrapleural, T1-5 LCSD is safe and effective for treating congenital LQTS in children, while minimizing the risks associated with VATS.

https://thejns.org/doi/abs/10.3171/2019.11.PEDS19424

KEYWORDS long QT syndrome; sympathectomy; cardiac sympathetic denervation; posterior approach

$\mathrm{P}$ EDIATRIC congenital cardiac arrhythmias, such as long QT syndrome (LQTS), provide a unique opportunity for neurosurgical intervention. In high-risk patients with LQTS whose condition is refractory to an implantable cardiac defibrillator (ICD) or medical management, left (or occasionally bilateral) cardiac sympathetic denervation (LCSD or CSD) is offered.,12,18

There are multiple approaches to LCSD and CSD, including thoracotomy and video-assisted thoracoscopic surgery (VATS). Institutions in which VATS is performed have reported a reduction in complication rates, shorter hospital and ICU stays, and a decrease in the risk of Horn- er syndrome when this technique is compared to thoracotomy. ${ }^{6,18}$ However, VATS requires single-lung ventilation during anesthesia, which increases the risk for postoperative hemothorax and pneumothorax. Furthermore, small children may not tolerate single-lung ventilation due to the need for placing a double-lumen endotracheal tube. ${ }^{8}$

Alternatively, a minimally invasive posterior LCSD has shown encouraging results. Three case series have demonstrated efficacy in removing the sympathetic ganglia via a posterior approach in 5 female adult patients with LQTS ${ }^{7}$ and 4 pediatric patients..$^{14,15}$

In this case report, we describe a posterior extrapleural

ABBREVIATIONS CSD = cardiac sympathetic denervation; ICD = implantable cardiac defibrillator; $L C S D=$ left CSD; LQTS = long QT syndrome; VATS = video-assisted thoracoscopic surgery; VF = ventricular fibrillation; VT = ventricular tachycardia.

SUBMITTED July 23, 2019. ACCEPTED November 14, 2019.

INCLUDE WHEN CITING Published online January 10, 2020; DOI: 10.3171/2019.11.PEDS19424. 
T1-5 LCSD in a pediatric patient with LQTS and ventricular fibrillation (VF)/ventricular tachycardia (VT) after a failed VATS LCSD due to respiratory insufficiency with single-lung ventilation, and we review the current literature for VATS and a posterior-approach LCSD for LQTS.

\section{Case Report}

History and Examination

The patient was a 9-year-old girl with a history of epilepsy and LQTS type III (SCN5A mutation), with a ventricular, single-chamber pacemaker placed at birth, upgraded to an ICD at the age of 2 years. She presented to the emergency department for generalized tonic-clonic seizures, followed by prolonged VF/VT and multiple ICD firings. Medical management with maximum doses of esmolol, lidocaine, and electrolyte optimization failed to stop her VF, with continued shocks from her ICD. She was subsequently sedated and intubated to halt catecholamine cascade. She was taken to the operating room for VATS LCSD 5 days later. However, she did not tolerate single-lung ventilation with a double-lumen endotracheal tube due to intraoperative respiratory insufficiency, so the LCSD was aborted. At this point, neurosurgery was consulted and a decision was made to proceed expediently with the minimally invasive posterior-approach sympathectomy.

\section{Operation}

In the operating room, the patient was placed in the prone position on the operating table and reintubated with a single-lumen endotracheal tube for standard dual-lung ventilation. Her respiratory insufficiency resolved. Defibrillator pads were placed, and her ICD was programmed for surgery. Fluoroscopy was used to identify the T1-5 vertebrae and costotransverse joints. Left paramedian incisions were marked, and the patient was then prepared and draped in a standard, sterile manner.

The first incision was made over the T1-2 costotransverse joints, and the thoracodorsal fascia was opened. Minimally invasive METRx serial dilating tubes were used to dilate up to a 22-mm diameter. Under microscopy, the $\mathrm{T} 1$ costotransverse joint was identified and dissected free of the surrounding soft tissues. The location was confirmed with navigation, and a costotransversectomy was performed with a 3-mm diamond burr. This surgical technique has been described in detail by Raskin et al. ${ }^{14,15} \mathrm{~A}$ schematic of the posterior-approach technique, as reported by Raskin et al.. ${ }^{14,15}$ can be seen in Fig. 1 .

During exploration of the inferior aspect of the scalene muscles underneath the $\mathrm{T} 1 \mathrm{rib}$ head, the most inferior portion of the brachial plexus was identified and avoided, and the cephalad caudad-oriented T1 sympathetic chain was subsequently identified. Great caution was taken to remain extrapleural. The chain was selected using a nerve hook, and the T1 section was cut with microscissors. The T2 sympathetic chain was visualized and sectioned in the same manner after angling the tubular retractor system caudad. This same minimally invasive approach was used for a sympathectomy of the T3-5 sympathetic chain via a more caudad paramedian incision. Hemostasis was achieved, and the incisions were closed in the typical layered fashion.

\section{Postoperative Course}

Following surgery, the patient was transferred to the pediatric ICU for observation. She remained on esmolol (400-500 $\mu \mathrm{g} / \mathrm{kg} / \mathrm{hr}$ ) throughout her stay and had no new episodes of VF/VT. Given the resolution of her VF/VT with only LCSD, it was decided that she did not require a right-sided CSD. She did have three 10-second episodes of stiffening and jerking, thought to be epileptic in nature according to the neurology department. These epileptic episodes were thought to be due to her doses of lidocaine $(50 \mu \mathrm{g} / \mathrm{kg} / \mathrm{min})$ for cardiac arrhythmias. She was started on Keppra (1000 mg twice daily) with plans for further workup with neurology as an outpatient. Otherwise, the patient recovered uneventfully from surgery and was discharged 12 days later.

\section{Follow-Up}

In the 2 years since hospitalization, the patient has had one additional episode of VT, which was attributed to electrolyte imbalance resulting from antibiotic-associated diarrhea at that time. Since then, with mexiletine $(250 \mathrm{mg}$ 3 times/day) and propranolol (160 mg 3 times/day), the patient has remained free of subsequent VF/VT and ICD shocks.

\section{Discussion}

We present the case of a 9-year-old girl with LQTS type III who presented in VF/VT with multiple ICD shocks; she was unable to undergo VATS LCSD due to intolerance of single-lung ventilation. Using standard dual-lung ventilation, minimally invasive T1-5 posterior extrapleural LCSD was performed and well tolerated without development of respiratory insufficiency, hemothorax, pneumothorax, or Horner syndrome. Immediately postoperatively, her episodes of VF/VT resolved while continuing medical therapy. During the 2-year follow-up period, she experienced a significant reduction in ICD shocks and had a single episode of VT in the setting of electrolyte abnormalities. This is the first case, to our knowledge, of successful conversion to posterior extrapleural LCSD from failed VATS due to respiratory insufficiency in a child with lifethreatening cardiac arrhythmia refractory to medical therapy and ICD placement.

\section{Anterior-Approach LCSD}

There are multiple approaches to performing LCSD, although anterior approaches via a thoracotomy, VATS LCSD, or the supraclavicular approach are most commonly used, with VATS LCSD being the gold standard approach. ${ }^{1,3,4,6,11-13,17-19}$ Anterior LCSD has demonstrated efficacy in children and adults with LQTS, with a reduction in cardiac events in up to $85 \%$ of patients. ${ }^{4,69,11-13,16}$

Although anterior-approach VATS has been the gold standard procedure for LCSD, it still has contraindications and significant risks. The VATS technique requires single-lung ventilation, which is difficult for specific patients to tolerate-namely, those with known pulmonary 

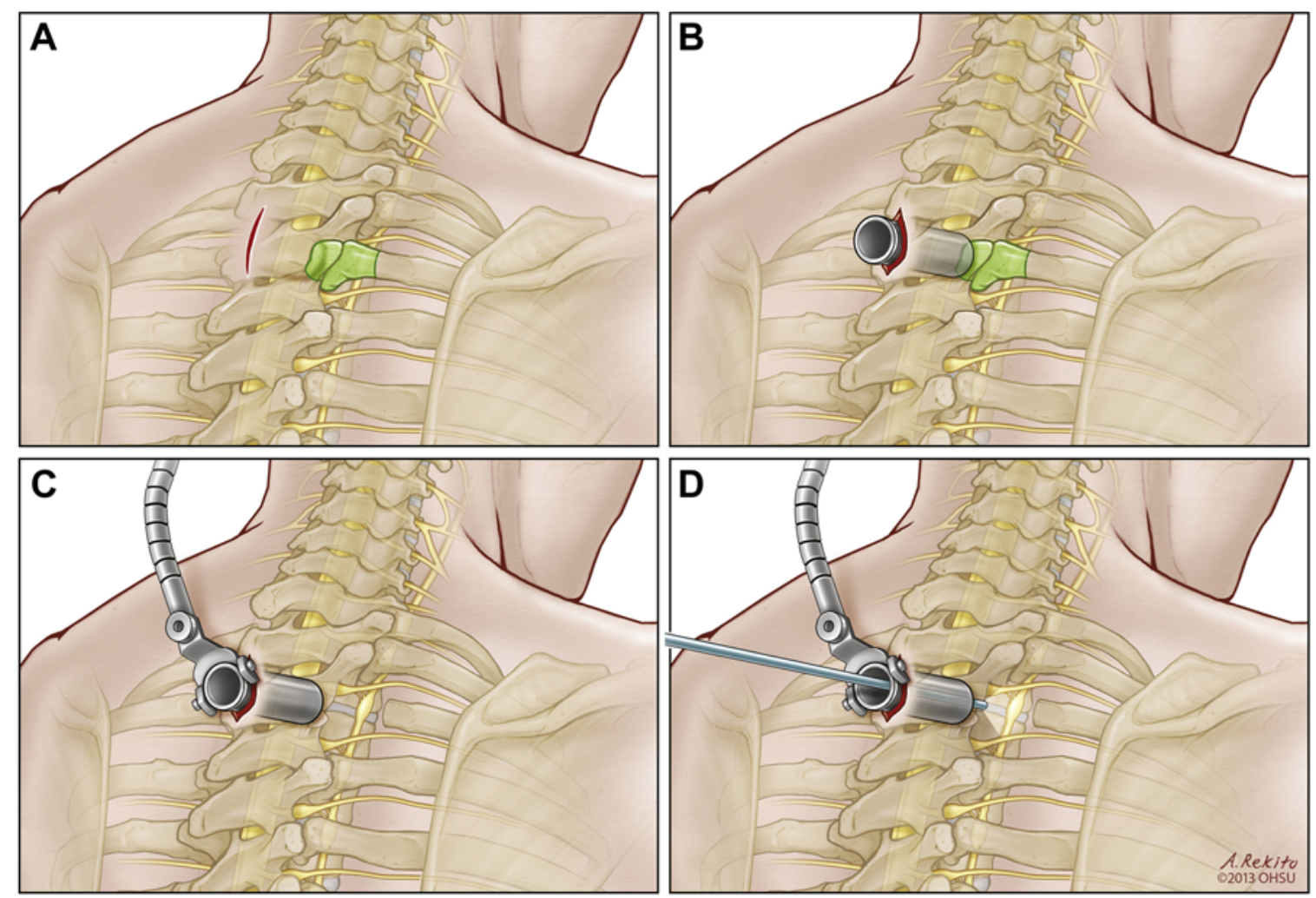

FIG. 1. The posterior extrapleural approach for CSD involves exposure of the T1-5 costotransverse joints (CTJs) using minimally invasive dilator tubes, so that costotransversectomy and subsequent sectioning of the underlying sympathetic ganglia can be performed. First, a paramedian incision is made over the fluoroscopically identified T1-5 CTJs (A). After the incision is made and thoracodorsal fascia is dissected, minimally invasive dilating tubes are used to expose the CTJs under the microscope (B), and mini-costotransversectomy is performed with a 3-mm diamond burr (C). The left and right T1-5 sympathetic ganglia are then sectioned and removed (D). Reprinted from World Neurosurgery, Volume 93, JS Raskin, JJ Liu, H Sun, A Nemecek, S Balaji, AM Raslan, Minimal access posterior approach for extrapleural thoracic sympathectomy: a cadaveric study and cases, p 490, Copyright 2016, with permission from Elsevier. Figure is available in color online only.

disease and small children due to the difficulty of placing a double-lumen endotracheal breathing tube in their small airways. ${ }^{8}$ Notably, single-lung ventilation also carries the significant risk of postoperative pneumothorax or hemothorax ${ }^{5,10}$ Furthermore, the supraclavicular approach causes left-sided Horner syndrome in $100 \%$ of patients due to disruption of ocular nerve fibers in or near the stellate ganglion. ${ }^{2}$ Lastly, anterior approaches inherently involve violation of the pleura to gain access to the sympathetic chain. This makes the posterior extrapleural approach appealing because it eliminates the need for single-lung ventilation, decreases the likelihood of Horner syndrome, and reduces the risk of pleural violation. ${ }^{7,14,15}$

\section{LCSD: The Posterior Approach}

Epstein et al. first described the posterior approach in 1996. In their study, an open procedure was performed in 5 patients with LQTS; it involved lateral retraction of the paraspinous muscles, T2-4 costotransversectomy, and sympathetic ganglion nerve stimulation with cardiac monitoring to identify all ganglia responsible for QT prolongation, $\mathrm{T}$ wave or $\mathrm{U}$ wave alterations, or the formation of ventricular arrhythmias. The ganglia associated with observed electrocardiogram changes, or ganglia at levels above it, were then removed. Postoperatively, all 5 patients showed a significant reduction in ventricular arrhythmias and syncopal symptoms. Three of the patients were free of ventricular arrhythmias at 32-month follow-up visits; 1 of the 3 symptom-free patients no longer required beta blockade or pacing. Importantly, no patient developed Horner syndrome, pneumothorax, or hemothorax postoperatively.

Gardner et al. ${ }^{8}$ proposed a minimally invasive endoscopic posterior approach in 2008 , in which a posterior sympathectomy was undertaken for the treatment of palmar hyperhidrosis in 2 patients. Tubular dilation, under fluoroscopic guidance, over the T3 costotransverse joint was used to visualize the sympathetic ganglia and underlying pleura. Visualization using the tube system was adequate for dissection of the pleura away from the sympathetic chain. Subsequent removal of the appropriate ganglia was achieved without causing pneumothorax or hemothorax. Both patients experienced resolution of their palmar hyperhidrosis following this procedure. One developed Horner syndrome postoperatively, thought to be due to a traction injury rather than injury as a result of the sympathectomy itself. 





In 2016, Raskin et al. published 2 studies, a cadaveric proof-of-concept study and case series, in which they used a minimally invasive, posterior, extrapleural LCSD under the microscope as a surgical approach in 2 children with medically refractory arrhythmias. ${ }^{14,15}$ In the cadaveric study, the same technique described by Gardner et al. ${ }^{8}$ was used on 5 fresh cadavers with normal spinal anatomy on both the left and right sides, for a total of 10 approaches. Bony depth, craniocaudal length, and width were measured to report parameters for adequate exposure of the sympathetic chain and occurrence of pleural violation. The authors found that the craniocaudal length was $11-17 \mathrm{~mm}$ and the width was $<18 \mathrm{~mm}$ (the diameter of the tubular retractors), the mediolateral drilling width was $12-18 \mathrm{~mm}$, and the mean drilling depth was $2.3 \mathrm{~cm}$. Pleural violation occurred in only 1 of the 10 cadaveric approaches..$^{14,15}$

Using the cadaveric information, posterior extrapleural LCSD was utilized in 2 medically refractory pediatric patients who had numerous prior ICD shocks: a 9-year-old girl with LQTS and a 13-year-old boy with hypertrophic cardiomyopathy. The girl with LQTS was free of cardiac events for 3 months postoperatively, but she required escalating beta blockade and needed numerous defibrillations by her ICD. She returned for a right-sided CSD 19 months later, which resulted in decreased defibrillation events. She did not develop pneumothorax or Horner syndrome with either surgery. The boy experienced a complete response to surgery and no longer had any defibrillation events while on beta blockade. He developed small bilateral apical pneumothoraces that resolved without chest tube placement. ${ }^{14}$ It was concluded that the minimally invasive, posterior, extrapleural LCSD was a safe and effective alternative approach for LCSD in children.

In summary, the aforementioned cases (Table 1) demonstrate the posterior minimally invasive LCSD approach is likely to reduce the development of Horner syndrome, eliminate the need for single-lung ventilation or pleural violation, and make LCSD a more tolerable procedure for children with LQTS..$^{14,15}$

\section{Limitations}

This case represents one child with LQTS who needed urgent conversion to a posterior LCSD due to her inability to tolerate single-lung ventilation. Generalizability is therefore limited. However, when the data from this case are combined with the data reported in other similar studies, it appears that the minimally invasive, posterior, extrapleural approach is safe and effective, including when used in urgent settings. A larger study would shed more light on this matter. This case highlights the approach as a useful alternative when VATS is not feasible. A comparative study between VATS and the minimally invasive posterior approach for LCSD would be helpful to compare efficacy and complication rates.

\section{Conclusions}

In pediatric patients with medically refractory LQTS who cannot tolerate single-lung ventilation during a sympathectomy in VATS, the minimally invasive posterior sympathectomy may provide an alternative approach to reduce cardiac events and treat arrhythmias. Furthermore, in emergency settings of respiratory insufficiency, conversion to a posterior sympathectomy can be done safely with low risk of postoperative complications.

\section{Acknowledgments}

We thank Shirley McCartney, $\mathrm{PhD}$, for editorial assistance.

\section{References}

1. Atallah J, Fynn-Thompson F, Cecchin F, DiBardino DJ, Walsh EP, Berul CI: Video-assisted thoracoscopic cardiac denervation: a potential novel therapeutic option for children with intractable ventricular arrhythmias. Ann Thorac Surg 86:1620-1625, 2008

2. Bhandari AK, Scheinman MM, Morady F, Svinarich J, Mason J, Winkle R: Efficacy of left cardiac sympathectomy in the treatment of patients with the long QT syndrome. Circulation 70:1018-1023, 1984

3. Bos JM, Bos KM, Johnson JN, Moir C, Ackerman MJ: Left cardiac sympathetic denervation in long QT syndrome: analysis of therapeutic nonresponders. Circ Arrhythm Electrophysiol 6:705-711, 2013

4. Chen L, Qin YW, Zheng CZ: Left cervicothoracic sympathetic ganglionectomy with thoracoscope for the treatment of idiopathic long QT syndrome. Int J Cardiol 61:1-3, 1997

5. Chiou TS, Chen SC: Intermediate-term results of endoscopic transaxillary T2 sympathectomy for primary palmar hyperhidrosis. Br J Surg 86:45-47, 1999

6. Collura CA, Johnson JN, Moir C, Ackerman MJ: Left cardiac sympathetic denervation for the treatment of long QT syndrome and catecholaminergic polymorphic ventricular tachycardia using video-assisted thoracic surgery. Heart Rhythm 6:752-759, 2009

7. Epstein AE, Rosner MJ, Hageman GR, Baker JH II, Plumb VJ, Kay GN: Posterior left thoracic cardiac sympathectomy by surgical division of the sympathetic chain: an alternative approach to treatment of the long QT syndrome. Pacing Clin Electrophysiol 19:1095-1104, 1996

8. Gardner PA, Ochalski PG, Moossy JJ: Minimally invasive endoscopic-assisted posterior thoracic sympathectomy. Neurosurg Focus 25(2):E6, 2008

9. He D, Costello JP, Nadler EP, Moak JP, Jonas RA, Nath DS: Left thoracoscopic sympathectomy used as primary therapy for a young child with intractable long QT syndrome. Pediatr Cardiol 34:1969-1971, 2013

10. Herbst F, Plas EG, Függer R, Fritsch A: Endoscopic thoracic sympathectomy for primary hyperhidrosis of the upper limbs. A critical analysis and long-term results of 480 operations. Ann Surg 220:86-90, 1994

11. Hofferberth SC, Cecchin F, Loberman D, Fynn-Thompson F: Left thoracoscopic sympathectomy for cardiac denervation in patients with life-threatening ventricular arrhythmias. J Thorac Cardiovasc Surg 147:404-409, 2014

12. Hwang SW, Thomas JG, Whitehead WE, Curry DJ, Dauser $\mathrm{RC}$, Kim ES, et al: Left thorascopic sympathectomy for refractory long QT syndrome in children. J Neurosurg Pediatr 8:455-459, 2011

13. Li J, Liu Y, Yang F, Jiang G, Li C, Hu D, et al: Video-assisted thoracoscopic left cardiac sympathetic denervation: a reliable minimally invasive approach for congenital long-QT syndrome. Ann Thorac Surg 86:1955-1958, 2008

14. Raskin JS, Liu JJ, Abrao A, Holste K, Raslan AM, Balaji S: Minimally invasive posterior extrapleural thoracic sympathectomy in children with medically refractory arrhythmias. Heart Rhythm 13:1381-1385, 2016 
15. Raskin JS, Liu JJ, Sun H, Nemecek A, Balaji S, Raslan AM: Minimal access posterior approach for extrapleural thoracic sympathectomy: a cadaveric study and cases. World Neurosurg 93:490.e1-490.e6, 2016

16. Reardon PR, Matthews BD, Scarborough TK, Preciado A, Marti JL, Conklin LD, et al: Left thoracoscopic sympathectomy and stellate ganglionectomy for treatment of the long QT syndrome. Surg Endosc 14:86, 2000

17. Schwartz PJ, Crotti L, Insolia R: Long-QT syndrome: from genetics to management. Circ Arrhythm Electrophysiol 5:868-877, 2012

18. Schwartz PJ, Priori SG, Cerrone M, Spazzolini C, Odero A, Napolitano C, et al: Left cardiac sympathetic denervation in the management of high-risk patients affected by the longQT syndrome. Circulation 109:1826-1833, 2004

19. Silver ES, Liberman L, Chung WK, Spotnitz HM, Chen JM, Ackerman MJ, et al: Long QT syndrome due to a novel mutation in SCN5A: treatment with ICD placement at 1 month and left cardiac sympathetic denervation at 3 months of age. J Interv Card Electrophysiol 26:41-45, 2009

\section{Disclosures}

The authors report no conflict of interest concerning the materials or methods used in this study or the findings specified in this paper.

\section{Author Contributions}

Conception and design: Raslan, Balaji. Acquisition of data: Blatt. Analysis and interpretation of data: Blatt. Drafting the article: Blatt, Cheaney, Holste. Critically revising the article: all authors. Reviewed submitted version of manuscript: all authors. Study supervision: Raslan.

\section{Correspondence}

Ahmed M. Raslan: Oregon Health \& Science University, Portland,OR.raslana@ohsu.edu. 